\title{
Determinação de um conjunto reduzido de características morfológicas para classificação de lesões mamárias em imagens de ultrassom utilizando máquinas de vetores de suporte
}

\author{
Carmina Dessana L. Nascimento ${ }^{1}$, Thales A. Silva1, Wagner C. A. Pereira ${ }^{2}$, Marly \\ G. F. Costa ${ }^{1}$, Cícero F. F. C. Filho ${ }^{1}$ \\ ${ }^{1}$ Centro de Pesquisa e Desenvolvimento de Tecnologia Eletrônica e da Informação - \\ Universidade Federal do Amazonas (CETELI/UFAM) \\ CEP 69077-000 - Manaus - AM - Brasil \\ ${ }^{2} \mathrm{PEB} / \mathrm{UFRJ}$ \\ \{cdessana, thales.tas, wcap58\} @gmail.com, \{mcosta, ccosta\} @ufam.edu.br
}

\begin{abstract}
The use of tools for computer-aided diagnosis (CAD) has been proposed for the detection and classification of breast cancer. Concerning breast cancer image diagnosis with ultrasound, some results found in literature show a better performance of morphological features on breast cancer lesion differentiating and that a reduced set of features shows a better performance than a large set of features. In this study, we evaluated the performance SVM classifiers with different kernels: polynomial and RBF. Different sets of morphological features were used for SVM training and classification. To select reduced sets of features, scalar selection technique with correlation was used. The best results obtained for accuracy and area under ROC curve were 92\% and 0.920 , respectively.
\end{abstract}

Resumo. $O$ uso de ferramentas de auxílio de diagnóstico (CAD) para detecção e classificação de câncer de mama tem sido difundido. No que diz respeito ao diagnóstico de câncer de mama por imagens de ultrassom, resultados encontrados na literatura revelam um melhor desempenho de classificação das lesões mamárias com características morfológicas e sugerem que um conjunto reduzido de características apresenta uma melhor performance de classificação em relação a um conjunto grande. Neste estudo foram avaliados o desempenho de classificadores SVM com diferentes kernels: polinomial e $R B F$. Conjuntos de características morfológicas distintos foram utilizados para treinamento e classificação. A seleção de conjuntos reduzidos de características, foi realizada através da técnica de seleção de escalar com correlação. Os melhores resultados obtidos para acurácia e AUC foram de $92 \%$ e 0.920 respectivamente

\section{Introdução}

O câncer de mama continua sendo a maior causa de mortes entre mulheres nos países desenvolvidos e em desenvolvimento [Who, 2014]. No Brasil, o câncer de mama ainda é a forma mais comum de câncer entre as mulheres, ocupando o primeiro lugar em incidência nas regiões Nordeste, Sul e Sudoeste, na proporção de 22,84\%, 24,14\% e $23,83 \%$, respectivamente. No Norte e Centro-Oeste, esta incidência é ultrapassada apenas pelo câncer do colo do útero [Sbcancerologia, 2015]. 
Na população mundial, a sobrevida média após cinco anos do diagnóstico é de $61 \%$, porém no Brasil, as taxas de mortalidade por câncer de mama continuam elevadas, muito provavelmente porque a doença ainda é diagnosticada principalmente em estágios avançados [INCa, 2015].

O diagnóstico precoce é importante para o tratamento do câncer e requer um procedimento confiável e preciso que possibilite o médico distinguir entre tumores malignos e benignos. Uma boa abordagem de diagnóstico produz tanto uma baixa taxa de falsos positivos (FP) quanto de falsos negativos (FN) [Cheng et al., 2010].

A mamografia é o exame de triagem utilizado amplamente na detecção precoce do câncer de mama, com um impacto fundamental na eficácia da terapia. No entanto, a sua especificidade é baixa e, como consequência, um número considerável de massas sólidas suspeitas é, geralmente, submetido a biópsia cirúrgica [Pereira et al., 2010]. Além de ser um procedimento invasivo e doloroso, a biópsia gera um estresse emocional no paciente [Zyout e Czajkowska, 2015]. Adicionalmente, a probabilidade de resultados positivos das lesões visualizadas na mamografia e encaminhadas para biópsia é baixa e gira em torno de $10 \%$ e $31 \%$.

Com o objetivo de melhorar o diagnóstico e reduzir o número de biópsias desnecessárias, a ultrassonografia (US) de mama emergiu como um importante complemento à mamografia, para pacientes com massa palpável, mamas densas e diagnósticos inconclusivos [Skaane, 1999]. Porém, a interpretação das imagens de ultrassonografia mamária (BUS) é subjetiva e dependente do expertise do médico ultrassonografista. Portanto, o diagnóstico diferencial das lesões de mama em imagens BUS deve ser realizado por um ultrassonografista altamente capacitado.

O uso de ferramentas que auxiliem o diagnóstico através de computador (CAD) vem sendo proposto para a detecção e classificação do câncer [Uniyal et al., 2013]. O CAD fornece informações importantes baseado em análises computacionais das imagens de ultrassom, para auxiliar os médicos tanto na localização das lesões quanto na classificação das mesmas como benignas ou malignas [Yeh et al., 2007].

Em geral, o desenvolvimento de sistemas de CAD para a detecção do câncer de mama envolve quatro etapas: pre-processamento. segmentação da imagem, seleção e extração de características, e a classificação propriamente dita [Cheng et al., 2010].

Ao mesmo tempo que representa as informações de forma adequada, um conjunto de características ótimo reduz a dimensão do vetor utilizado para treinamento e teste do classificador [Theodoridis e Koutroumbas, 2008]. Assim, em sistemas CAD para detecção de câncer de mama, é essencial a etapa de extração e seleção de características [Wu e Moon, 2008].

No caso de classificação de lesões em BUS, a literatura tem mostrado que características relacionadas a textura e morfologia das lesões segmentadas tem sido muito utilizadas para classificar os nódulos detectados [Zhou et al., 2013].

Com o objetivo de encontrar o menor conjunto de características de forma a produzir uma efetiva melhora no desempenho da classificação, Flores et al (2015) avaliaram um conjunto de características morfológicas e de textura, propostas anteriormente na literatura. Após análise, os autores sugeriram o uso de apenas cinco características morfológicas para classificar lesões mamárias. 
$\mathrm{Na}$ etapa de classificação dos nódulos, as Máquinas de Vetores de Suporte (SVM) tem sido usadas extensivamente [Huang et al., $2007 \mathrm{Wu}$ e Moon, 2008].

Neste trabalho, são investigadas as características morfológicas das lesões compiladas por Flores et al. (2015). Utilizou-se técnicas de seleção escalar de variáveis para reduzir o referido conjunto de características morfológicas. O método de classisficação utilizado foi SVM, associado a diferentes kernels. Combinações diversas de características e de kernels foram empregadas, no sentido de otimizar a taxa de classificação das lesões como benignas ou malignas.

\section{Materiais e Métodos}

\subsection{Materiais}

O banco de dados utilizado neste trabalho é constituído de características relativas aos contornos de lesões, oriundas de 100 imagens BUS, de 100 pacientes. As imagens foram adquiridas no Instituto Nacional de Câncer ( $\mathrm{INCa}$ ), durante rotinas de diagnóstico utilizando o equipamento Sonoline-Sienna (Siemens ${ }^{\circledR}$ ) com transdutores de $7.5 \mathrm{MHz}$ e resolução lateral e axial de $0.49 \mathrm{~mm}$ e $0.45 \mathrm{~mm}$ respectivamente. Foram armazenadas em formato JPG, com 8 bits de resolução de profundidade. Na Figura 1 são apresentados exemplos de imagens BUS do referido banco de dados.

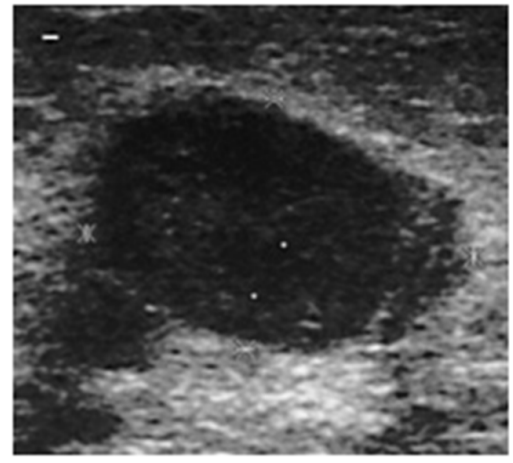

(a)

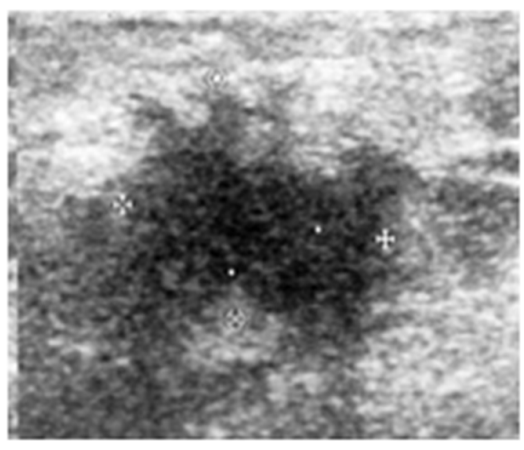

(b)

Figura 1. Exemplo de imagens BUS do banco de dados utilizado: (a) BUS com lesão benigna; (b) BUS com lesão maligna

Os pacientes foram submetidos a exames histopatológicos a fim de verificar seguramente a natureza das lesões encontradas nas imagens. Foram identificadas 50 imagens com lesões de natureza maligna e 50 imagens com lesões de natureza benigna.

A região de interesse (ROI) das imagens foi delineada manualmente por dois radiologistas experientes, compreendendo a lesão e a área de fundo na sua vizinhança, que carrega informações importantes para a rotina de diagnóstico por ultrassom. Além disso, cada ROI foi segmentada utilizando um procedimento de contorno semiautomático (SAC), baseado em operadores morfológicos.

Após a segmentação, obteve-se uma imagem binária resultante que descreve e representa a imagem original de forma adequada para o processo de classificação, de onde foram extraídas 22 características relativas ao contorno das lesões, sendo duas relacionadas ao esqueleto morfológico: Elliptic Normalized Skeleton (ENS) e Skeleton end $\left(S_{\#}\right)$; seis ao comprimento radial normalizado: razão da área $\left(d_{A}\right)$, média $\left(d_{\mu}\right)$, desvio 
padrão $\left(d_{\sigma}\right)$, a entropia do histograma $\left(d_{E}\right)$, o índice de rugosidade $\left(d_{R}\right)$, e zero-crossing $\left(d_{Z}\right)$; nove ao polígono convexo: Overlap ratio (RS), Normalized residual value (NRV), Number of protuberances and depressions (NSPD), Lobulation index (LI), Proportional distance (PD), Convexity (Cnvx), Elliptic normalized circumference (ENC), Distância de Hausdorff (HD) e Average distance (MD); quatro relacionadas a circularidade: Orientation $\left(O_{E}\right)$, Circularity $A\left(C_{a}\right)$, Circularity $B\left(C_{b}\right)$ e Circularity $C\left(C_{c}\right)$, e uma relacionada a elipse equivalente: Depth-to-width ratio (DWR) [Flores, 2009].

$\mathrm{O}$ vetor de características de contorno é então definido por $\mathcal{D}=\{E N S$, $S_{\#}, d_{\mu}, d_{\sigma}, d_{E}, d_{A}, d_{Z}, d_{R}, R S, N R V, N S P D, L I, P D, C n v x, E N C, H D, M D, O_{E}, C_{a}, C_{b}, C_{c} \quad e$ $D W R\}$.

\subsection{Métodos}

A classificação das lesões é realizada segundo o diagrama em blocos da Figura 2. As principais etapas do método são: seleção de características, separação dos dados e classificação. Essas três etapas são descritas a seguir.

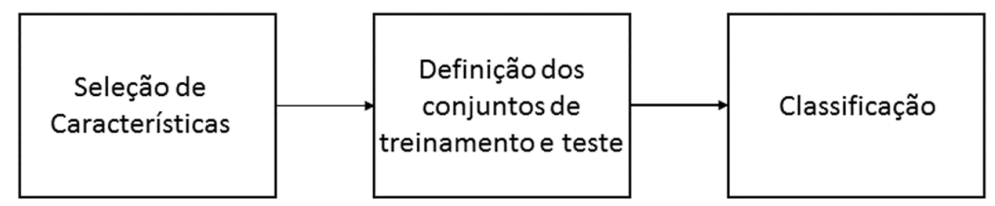

Figura 2. Etapas do método de classificação das lesões

\subsubsection{Seleção de Características}

A fim de determinar quais características, dentre as disponíveis no banco de dados, possuem melhor representatividade, foi utilizada a técnica de seleção escalar de características.

Nesta técnica é utilizado um critério de medição de separação das classes, como por exemplo, curva ROC (Receiver Operator Characteristic) e divergência unidimensional ou FDR (Fisher's Discriminant Ratio) [Theodoridis e Koutroumbas, 2008]. Este último critério foi utilizado neste trabalho e é definido como:

$$
F D R=\frac{\left(\mu_{1}-\mu_{2}\right)^{2}}{\sigma_{1}^{2}+\sigma_{2}^{2}}
$$

em que, $\mu_{1}$ e $\mu_{2}$ são as médias e $\sigma_{1}$ e $\sigma_{2}$ são os desvios padrões das classes $\omega_{1}$ e $\omega_{2}$ respectivamente.

Calcula-se inicialmente a medida FDR $(C(k)))$ para cada característica $x_{k}$. A que apresenta maior valor é selecionada e denominada de $x_{i l}$. Define-se então, o coeficiente de correlação cruzada entre duas características $i$ e $j$ como:

$$
\rho_{i j}=\frac{\sum_{n=1}^{N} x_{n i} x_{n j}}{\sqrt{\sum_{n=1}^{N} x_{n i}^{2} \sum_{n=1}^{N} x_{n j}^{2}}}
$$


em que, $n=1,2, \ldots, N$ e $k=1,2, \ldots, m, x_{n k}$ é a k-ésima característica do n-ésimo padrão.O $\rho_{i j}$ é obtido entre a primeira característica selecionada $\left(x_{i 1}\right)$ e as $m-1$ restantes.

A segunda característica selecionada $\left(x_{i 2}\right)$ é obtida de forma que:

$$
i_{2}=\arg \max _{j}\left\{\alpha_{1} C(j)-\alpha_{2}\left|\rho_{i_{1} j}\right|\right\} \text { para todo } j \neq i
$$

em que, $\alpha_{1}$ e $\alpha_{2}$ são fatores que regulam a importância de cada termo. Neste trabalho, os valores utilizados foram $\alpha_{1}=\alpha_{2}=0,5$.

As demais características $\left(x_{i k}\right.$, em que $\left.k=3, \ldots, m\right)$ são selecionadas de forma que:

$$
i_{k}=\arg \max _{j}\left\{\alpha_{1} C(j)-\frac{\alpha_{2}}{k-1} \sum_{r=1}^{k-1}\left|\rho_{i_{r j}}\right|\right\}
$$

\subsubsection{Definição dos Conjuntos de Treinamento e Teste e Classificação}

$\mathrm{Na}$ etapa de classificação, as lesões de uma imagem são classificadas como malignas ou benignas. O método de classificação empregado foi o SVM. A divisão do conjunto de dados em treinamento e teste foi realizada através da técnica K-Fold Cross Validation, $\operatorname{com} k=4$.

\subsubsection{K-FoldCross Validation}

No $k$-fold cross-validation, ou validação cruzada, o conjunto de dados $\mathcal{D}$, é dividido aleatoriamente em $k$ subconjuntos mutuamente exclusivos $\mathcal{D}_{1}, \mathcal{D}_{2}, \ldots, \mathcal{D}_{\mathrm{k}}$, de tamanhos aproximadamente iguais. Um grupo é excluído e o classificador é treinado com os $k-1$ conjuntos restantes. Posteriormente, o classificador é testado no conjunto previamente excluído. Esse processo é repetido $k$ vezes, até que todos os conjuntos tenham sido utilizados para teste [Kohavi, 1995].

A estimativa de acurácia na validação cruzada é o número total de classificações corretas, dividido pelo número de instâncias do conjunto de dados.

Neste trabalho, o conjunto de dados foi dividido em 4 grupos, cada um com 25 vetores de características. Os grupos possuem alternadamente 13 vetores referentes a lesões com resultado histopatológico de malignidade e 12 referentes a lesões com resultado histopatológico de benignidade.

\subsubsection{Máquina de Vetores de Suporte}

SVM são classificadores binários que separam duas classes utilizando um hiperplano que maximiza a margem de separação [Haykin, 2009], conforme ilustrado na Figura 3. 


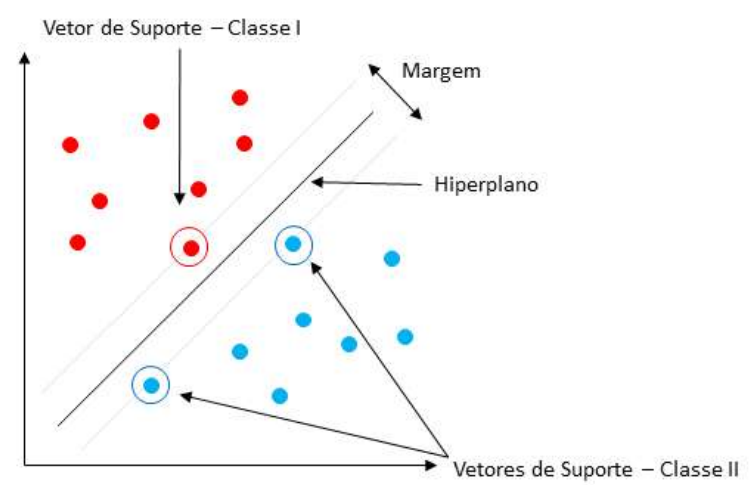

Figura 3. llustração de hiperplano ideal para Máquinas de Vetores de Suporte

De acordo com Theodoridis e Koutroumbas (2008), para classes separáveis, os parâmetros do hiperplano que maximizam a margem são calculados através da determinação do vetor de pesos $w$ e da polarização $w_{0}$, tal que a expressão (5) seja minimizada satisfazendo as condições de Karush-Kuhn-Tucker (KKT).

$$
J(w)=\frac{1}{2}\|w\|^{2}
$$

Por outro lado, para classes não separáveis são utilizadas variáveis de folga $\left(\xi_{i}\right)$. Desta forma os parâmetros podem ser obtidos minimizando-se a expressão (6), com o objetivo de maximizar a margem, mantendo o valor de pontos em que $\xi<0$, o mínimo possível.

$$
J\left(w, w_{0}, \xi\right)=\frac{1}{2}\|w\|^{2}+C \sum_{i=1}^{N} \xi_{i}
$$

em que o parâmetro $\mathrm{C}$ é uma constante positiva que relaciona a variável de folga e a margem.

Para separação de classes de forma não linear, as máquinas de vetores de suporte utilizam kernels para mapear o vetor de características para espaços com dimensões maiores. Neste trabalho, foram utilizados os kernels RBF (Radial Basis Function) e polinomial.

Foram realizadas simulações com cada conjunto de características obtido através do método de seleção escalar, além do conjunto inicial que compreende as 22 características, utilizando os kernels supracitados, variando suas ordens de 1 a 5 .

\section{Resultados}

\subsection{Conjunto de Características Selecionadas}

A seleção das melhores características foi feita a partir do conjunto de características descrito previamente na seção 2.1. Utilizando a técnica de seleção escalar, conjuntos com $2,3,4,5,6,7,8,9,10,11$ e 12 características foram selecionados. A Tabela 1 mostra as características selecionadas para cada um desses conjuntos. 
Tabela 1. Conjuntos de características selecionadas

\begin{tabular}{|c|l|}
\hline Número de características & \multicolumn{1}{|c|}{ Conjunto de características $\left(\mathcal{D}_{s}\right)$} \\
\hline 2 & $\mathrm{C}_{\mathrm{nvx}}, \mathrm{LI}$ \\
\hline 3 & $\mathrm{C}_{\mathrm{nvx}}, \mathrm{LI}, \mathrm{ENS}$ \\
\hline 4 & $\mathrm{C}_{\mathrm{nvx}}, \mathrm{LI}, \mathrm{ENS}, \mathrm{PD}$ \\
\hline 5 & $\mathrm{C}_{\mathrm{nvx}}, \mathrm{LI}, \mathrm{ENS}, \mathrm{PD}, \mathrm{ENC}$ \\
\hline 6 & $\mathrm{C}_{\mathrm{nvx}}, \mathrm{LI}, \mathrm{ENS}, \mathrm{PD}, \mathrm{ENC}, \mathrm{DWR}$ \\
\hline 7 & $\mathrm{C}_{\mathrm{nvx}}, \mathrm{LI}, \mathrm{ENS}, \mathrm{PD}, \mathrm{ENC}, \mathrm{DWR}, \mathrm{MD}$ \\
\hline 8 & $\mathrm{C}_{\mathrm{nvx}}, \mathrm{LI}, \mathrm{ENS}, \mathrm{PD}, \mathrm{ENC}, \mathrm{DWR}, \mathrm{MD}, \mathrm{NRV}$ \\
\hline 9 & $\mathrm{C}_{\mathrm{nvx}}, \mathrm{LI}, \mathrm{ENS}, \mathrm{PD}, \mathrm{ENC}, \mathrm{DWR}, \mathrm{MD}, \mathrm{NRV}, \mathrm{NSPD}$ \\
\hline 10 & $\mathrm{C}_{\mathrm{nvx}}, \mathrm{LI}, \mathrm{ENS}, \mathrm{PD}, \mathrm{ENC}, \mathrm{DWR}, \mathrm{MD}, \mathrm{NRV}, \mathrm{NSPD}, \mathrm{S}_{\#}$ \\
\hline 11 & $\mathrm{C}_{\mathrm{nvx}}, \mathrm{LI}, \mathrm{ENS}, \mathrm{PD}, \mathrm{ENC}, \mathrm{DWR}, \mathrm{MD}, \mathrm{NRV}, \mathrm{NSPD}, \mathrm{S}_{\#,}, \mathrm{HD}$ \\
\hline 12 & \begin{tabular}{l}
$\mathrm{C}_{\mathrm{nvx}}, \mathrm{LI}, \mathrm{ENS}, \mathrm{PD}, \mathrm{ENC}, \mathrm{DWR}, \mathrm{MD}, \mathrm{NRV}, \mathrm{NSPD}, \mathrm{S}_{\#,}, \mathrm{HD}$, \\
\hline
\end{tabular} \\
\hline
\end{tabular}

Observa-se que subconjunto com as cinco melhores características, $\{C n v x, L I, E N S, P D, E N C\}$ apresenta grande diferença em relação ao conjunto de melhores características, $\mathcal{D}_{c}$, sugerido por Flores et al. (2015), quais sejam, $\left\{E N S, N S P D, D W R, R S, O_{E}\right\}$. A característica Orientation, $O_{E}$, contida em $\mathcal{D}_{c}$, não pertence ao conjunto de 12 características, $\mathcal{D}_{S}$ obtido. Além disso, as características $R S$, e NSPD pertecem apenas aos sub-conjuntos com mais de 5 características.

\subsection{Classificação}

Para cada simulação com uma combinação diferente de conjunto de características, kernel e ordem do kernel, foram calculadas a acurácia e área sobre a curva ROC, AUC. A semelhança entre estes valores deve-se ao fato da saída do classificador SVM ser binária, ou seja, diferentemente das redes neurais, não é possível variar um limiar de decisão para obter diferentes valores de sensibilidade e especificidade. Desta forma, a curva ROC é constituída de um único ponto e a área sobre a curva ROC coincide com o valor da acurácia.

A Tabela 2 mostra os resultados obtidos com o kernel polinomial e a Tabela 3 com o kernel RBF. Em cada tabela é mostrado o desempenho do classificador SVM em termos da acurácia (Acr) e AUC, para cada subconjunto utilizado de 1, 2, 3, 4, 5, 6, 7, 8, $9,10,11,12$ e 22 características. A ordem dos kernels variou de 1 a 5.

A partir da Tabela 2 observa-se que o melhor resultado obtido para o kernel polinomial, foi uma acurácia de $92 \%$ e AUC de 0.920 . Esse resultado foi alcançado utilizando-se subconjuntos com 10, 11 e 12 características e com a ordem kernel igual a 1. As simulações com valores de ordem do kernel maiores que 1 apresentaram valores de acurácia e AUC significantemente menores.

O melhor desempenho do classificador SVM com kernel RBF, acurácia de 92\% e AUC de 0.920, foi obtido utilizando-se subconjuntos com 6 e 7 características e com a ordem do kernel igual a 1, como indicado na Tabela 3. Afirma-se ser este o melhor resultado por corresponder aos melhores valores de acurácia e AUC obtidos, com o menor subconjunto de características. 
Tabela 2. Resultados com Kernel Polinomial

\begin{tabular}{|c|c|c|c|c|c|c|c|c|c|c|c|}
\hline \multirow{2}{*}{\multicolumn{2}{|c|}{$\begin{array}{c}\text { Ordem } \\
\begin{array}{c}\text { Medida de } \\
\text { desempenho }\end{array}\end{array}$}} & \multicolumn{2}{|c|}{1} & \multicolumn{2}{|c|}{2} & \multicolumn{2}{|c|}{3} & \multicolumn{2}{|c|}{4} & \multicolumn{2}{|c|}{5} \\
\hline & & \multirow{2}{*}{$\begin{array}{c}\text { Acr (\%) } \\
76 \\
\end{array}$} & \multirow{2}{*}{$\begin{array}{r}\text { AUC } \\
0,76 \\
\end{array}$} & \multirow{2}{*}{$\begin{array}{c}\text { Acr (\%) } \\
83 \\
\end{array}$} & \multirow{2}{*}{$\begin{array}{r}\text { AUC } \\
0,83 \\
\end{array}$} & \multirow{2}{*}{$\begin{array}{c}\text { Acr (\%) } \\
85 \\
\end{array}$} & \multirow{2}{*}{$\begin{array}{r}\text { AUC } \\
0,85 \\
\end{array}$} & \multirow{2}{*}{$\begin{array}{c}\text { Acr (\%) } \\
84 \\
\end{array}$} & \multirow{2}{*}{$\begin{array}{r}\text { AUC } \\
0,84 \\
\end{array}$} & \multirow{2}{*}{\begin{tabular}{|c|} 
Acr (\%) \\
74 \\
\end{tabular}} & \multirow{2}{*}{$\begin{array}{r}\text { AUC } \\
0,74 \\
\end{array}$} \\
\hline \multirow{13}{*}{ 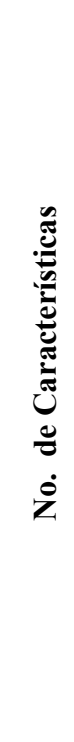 } & 1 & & & & & & & & & & \\
\hline & 2 & 87 & 0,87 & 76 & 0,76 & 75 & 0,75 & 75 & 0,75 & 83 & 0,83 \\
\hline & 3 & 85 & 0,85 & 85 & 0,85 & 84 & 0,84 & 78 & 0,78 & 77 & 0,77 \\
\hline & 4 & 87 & 0,87 & 86 & 0,86 & 83 & 0,83 & 81 & 0,81 & 80 & 0,80 \\
\hline & 5 & 87 & 0,87 & 82 & 0,82 & 83 & 0,83 & 76 & 0,76 & 76 & 0,76 \\
\hline & 6 & 90 & 0,90 & 88 & 0,88 & 83 & 0,83 & 83 & 0,83 & 84 & 0,84 \\
\hline & 7 & 90 & 0,90 & 85 & 0,85 & 82 & 0,82 & 83 & 0,83 & 81 & 0,81 \\
\hline & 8 & 90 & 0,90 & 86 & 0,86 & 81 & 0,81 & 82 & 0,82 & 80 & 0,80 \\
\hline & 9 & 91 & 0,91 & 85 & 0,85 & 84 & 0,84 & 82 & 0,82 & 81 & 0,81 \\
\hline & 10 & 92 & 0,92 & 86 & 0,86 & 80 & 0,80 & 80 & 0,80 & 78 & 0,78 \\
\hline & 11 & 92 & 0,92 & 87 & 0,87 & 76 & 0,76 & 72 & 0,72 & 74 & 0,74 \\
\hline & 12 & 92 & 0,92 & 85 & 0,85 & 76 & 0,76 & 72 & 0,72 & 75 & 0,75 \\
\hline & 22 & 89 & 0,89 & 85 & 0,85 & 85 & 0,85 & 80 & 0,80 & 80 & 0,80 \\
\hline
\end{tabular}

Tabela 3. Resultados com Kernel RBF

\begin{tabular}{|c|c|c|c|c|c|c|c|c|c|c|c|}
\hline \multirow{2}{*}{\multicolumn{2}{|c|}{$\begin{array}{c}\text { Sigma } \\
\begin{array}{r}\text { Medida de } \\
\text { desempenho }\end{array}\end{array}$}} & \multicolumn{2}{|c|}{1} & \multicolumn{2}{|c|}{2} & \multicolumn{2}{|c|}{3} & \multicolumn{2}{|c|}{4} & \multicolumn{2}{|c|}{5} \\
\hline & & \multirow{2}{*}{\begin{tabular}{|c|}
$\operatorname{Acr}(\%)$ \\
86 \\
\end{tabular}} & \multirow{2}{*}{$\begin{array}{r}\text { AUC } \\
0,86 \\
\end{array}$} & \multirow{2}{*}{$\begin{array}{c}\text { Acr (\%) } \\
85 \\
\end{array}$} & \multirow{2}{*}{$\begin{array}{r}\text { AUC } \\
0,85 \\
\end{array}$} & \multirow{2}{*}{$\begin{array}{c}\text { Acr (\%) } \\
69 \\
\end{array}$} & \multirow{2}{*}{$\begin{array}{r}\text { AUC } \\
0,68 \\
\end{array}$} & \multirow{2}{*}{$\begin{array}{c}\text { Acr (\%) } \\
77 \\
\end{array}$} & \multirow{2}{*}{$\begin{array}{r}\text { AUC } \\
0,77 \\
\end{array}$} & \multirow{2}{*}{\begin{tabular}{|c|} 
Acr (\%) \\
73 \\
\end{tabular}} & \multirow{2}{*}{$\begin{array}{r}\text { AUC } \\
0,73 \\
\end{array}$} \\
\hline \multirow{13}{*}{ 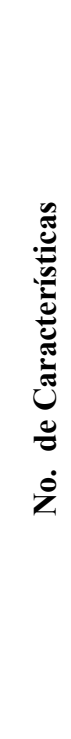 } & 1 & & & & & & & & & & \\
\hline & 2 & 87 & 0,87 & 87 & 0,87 & 87 & 0,87 & 87 & 0,87 & 88 & 0,88 \\
\hline & 3 & 84 & 0,84 & 86 & 0,86 & 87 & 0,87 & 85 & 0,85 & 85 & 0,85 \\
\hline & 4 & 89 & 0,89 & 88 & 0,88 & 88 & 0,88 & 88 & 0,88 & 88 & 0,88 \\
\hline & 5 & 88 & 0,88 & 86 & 0,86 & 87 & 0,87 & 87 & 0,87 & 88 & 0,88 \\
\hline & 6 & 92 & 0,92 & 91 & 0,91 & 90 & 0,90 & 89 & 0,89 & 90 & 0,90 \\
\hline & 7 & 92 & 0,92 & 91 & 0,91 & 90 & 0,90 & 89 & 0,89 & 89 & 0,89 \\
\hline & 8 & 90 & 0,90 & 90 & 0,90 & 89 & 0,89 & 88 & 0,88 & 87 & 0,87 \\
\hline & 9 & 91 & 0,91 & 90 & 0,90 & 89 & 0,89 & 88 & 0,88 & 87 & 0,87 \\
\hline & 10 & 91 & 0,91 & 91 & 0,91 & 89 & 0,89 & 89 & 0,89 & 88 & 0,88 \\
\hline & 11 & 91 & 0,91 & 89 & 0,89 & 89 & 0,89 & 88 & 0,88 & 88 & 0,88 \\
\hline & 12 & 90 & 0,90 & 89 & 0,89 & 89 & 0,89 & 89 & 0,89 & 88 & 0,88 \\
\hline & 22 & 81 & 0,81 & 87 & 0,87 & 88 & 0,88 & 88 & 0,88 & 88 & 0,88 \\
\hline
\end{tabular}


Nota-se que o desempenho dos classificadores varia não só com o subconjunto de características escolhido, mas também com o kernel utilizado no SVM. Entretanto, ambas tabelas confirmam que a seleção escalar de características realizada impactaram positivamente o desempenho do classificador.

\section{Discussão e Conclusão}

Comparando os resultados mostrados nas Tabelas 2 e 3, pode-se observar que a redução do conjunto de características realizada através da técnica de seleção escalar proporciona uma significante melhora em relação ao uso das 22 características.

Enquanto que, quando o kernel polinomial é utilizado, os resultados obtidos com os subconjuntos com 6, 7 e 8 características são moderadamente superiores ao resultado obtido utilizando-se o conjunto completo de 22 características, com o kernel RBF todos os subconjuntos de características apresentam um desempenho melhor em relação ao conjunto completo, destacando-se que os melhores resultados foram obtidos com os subconjuntos de 6 e 7 características.

Portanto, neste trabalho sugerimos o uso do seguinte conjunto de 6 características para a classificação de lesões em imagens de ultrassom mamárias: convexity, lobulation index, proportional distance, elliptic normalized skeleton, elliptic normalized circumference e depth-to-width ratio. Este subconjunto apresentou resultados em termos de acurácia e AUC superiores aos resultados obtidos utilizando-se o conjunto total de características independente do kernel utilizado

Utilizando o critério minimal-redundancy-maximal-relevance (mrMR), baseado em informação mútua (MI), Flores et al. (2015) propôs o uso do seguinte conjunto de 5 características para classificação das lesões em imagens BUS: elliptic normalized skeleton, orientation, number of protuberances and depressions, depth-to-width ratio and overlap ratio. Comparando os dois conjuntos notamos que existe a interseção de apenas duas características: elliptic normalized skeleton, e depth-to-width ratio. Os resultados obtidos pelos autores utilizando local fisher discriminant analysis (LFDA) para classificação com o conjunto supracitado foram AUC de 0.942, valores superiores aos obtidos neste trabalho. Esta diferença é influenciada tanto pelo método de seleção de características e classificador utilizado quanto pela quantidade de amostras disponíveis para o treinamento do classificador.

Utilizando SVM para classificação de lesões em imagens de ultrassom mamárias Huang et al. (2006) a partir de um conjunto de 118 imagens BUS com lesões histopatologicamente confirmadas obtiveram AUC de 0.91 com um conjunto inicial de 19 características e AUC 0.90, quando o conjunto foi reduzido a 6 características utilizando a técnica PCA. Com um conjunto inicial de 210 imagens, Wu e Moon (2008) obtiveram $90.95 \%$ de acurácia e com um subconjunto de 6 características $92.86 \%$. Portanto, os resultados apresentados, confirmam que a redução do conjunto de características é de extrema importância para o desempenho do classificador.

\section{Referências}

Cheng, H. D. et al. Automated breast cancer detection and classification using ultrasound images: A survey. Pattern Recognition, v. 43, n. 1, p. 299-317, 2010. 
Flores, W. G. Desarrollo de una Metodología Computacional para la Clasificación de Lesiones de Mama en Imágenes Ultrasónicas. 2009. Departamento de Ingeniería Eléctrica Sección de Bioelectrónica, Instituto Politécnico Nacional

Flores, W. G.; Pereira, W. C. D. A.; Infantosi, A. F. C. Improving classification performance of breast lesions on ultrasonography. Pattern Recognition, v. 48, n. 4, p. 1125 - 1136, 2015.

Haykin, S. S. Neural Networks and Learning Machines. 2009.

Huang, Y. L.; Wang, K. L.; Chen, D. R. Diagnosis of breast tumors with ultrasonic texture analysis using support vector machines. Neural Computing \& Applications, v. 15, n. 2, p. 164-169, 2006.

INCA. Mama. p. Câncer de Mama, 2015. Disponível em: < (http://www2.inca.gov.br/wps/wcm/connect/tiposdecancer/site/home/mama/cancer_ mama $>$.

Kohavi, R. A Study of Cross-Validation and Bootstrap for Accuracy Estimation and Model Selection. International Joint Conference on Artificial Intelligence, v. 2, p. 1137-1143, 1995.

Pereira, W. C. A. et al. A non-linear morphometric feature selection approach for breast tumor contour from ultrasonic images. Computers in Biology and Medicine, 2010.

SBCANCEROLOGIA. Cancer de Mama. 2015. Disponível em: < http://www.sbcancer.org.br/home2/site/index.php?option=com_content\&view=articl e\&id=110: cancer-de-mama\&catid=29\&Itemid=123 >. Acessoem: 02/12/2015.

Skaane, P. Ultrasonography as adjunct to mammography in the evaluation of breast tumors. Actaradiologica. Supplementum, 1999

Theodoridis, S.; Koutroumbas, K. Pattern Recognition, Fourth Edition. 4th. Academic Press, 2008.

Uniyal, N. et al. A new approach to ultrasonic detection of malignant breast tumors. 2013 Ieee International Ultrasonics Symposium (Ius), p. 96-99, 2013

WHO. Breast cancer: prevention and control. 2014. Disponível em: < http://www.who.int/cancer/detection/breastcancer/en/index $4 . h t m l>$.

Wu, W.-J.; Moon, W. K. Ultrasound breast tumor image computer-aided diagnosis with texture and morphological features. Academic Radiology, v. 15, n.7, p. 873-880, 2008.

Yeh, C.-K. et al. Automatic ultrasonic breast lesions detection using support vector machine based algorithm. Medical Imaging: Ultrasonic Imaging and Signal Processing, p.51317-51317, 2007.

Zhou, S. et al. Shearlet-based texture feature extraction for classification of breast tumor in ultrasound image. Biomedical Signal Processing and Control, v. 8, n. 6, p. 688-696, 2013.

Zyout, I.; Czajkowska, J., Marcin. Multi-scale Textural Feature Extraction and Particle Swarm Optimization Based Model Selection for False Positive Reduction in Mammography. Computerized Medical Imaging and Graphics, 2015. 